\section{Ejaculation training, seminal} alkaline phosphatase and semen preservation through cooling in a milk-based extender in domestic cats
Journal of Feline Medicine and Surgery 2014, Vol. 16(4) 312-316

(C) ISFM and AAFP 2013

Reprints and permissions:

sagepub.co.uk/journalsPermissions.nav DOI: $10.1177 / 1098612 \times 13508192$ jfms.com

\author{
Carla Valiente ${ }^{1}$, Pablo E de la Sota ${ }^{1}$, Sandra Arauz ${ }^{2}$ \\ and Cristina Gobello ${ }^{1}$
}

\begin{abstract}
The purpose of this report is to describe (1) the training of domestic cats in ejaculation into an artificial vagina (AV), (2) alkaline phosphatase (AP) concentrations in whole ejaculates, and (3) the in vitro effect of a skimmed-milk plus egg yolk (SM-Y) extender on feline spermatozoa incubated at $4^{\circ} \mathrm{C}$. Five post-pubertal cats were trained to ejaculate into an AV three times a week for 20 mins in the presence of a teaser queen. Fifty AV-obtained ejaculates were macro- and microscopically assessed, and the AP therein measured by optimized colorimetry. Eighty AV-obtained ejaculates were pooled, diluted in SM-Y extender [80\% (v/v) skimmed milk, 20\% (v/v) egg yolk, and antibiotics], stored at $4^{\circ} \mathrm{C}$ and evaluated daily for 6 days. All the animals could be trained to ejaculate, although the interval up to the first AV ejaculation varied from 1.5 to 5.5 months (mean 3.9 months). The final performance at collection ranged from excellent to poor and was inversely related to the training period required in all cases. The mean AP concentration in whole ejaculates was 20,645.6 \pm 4405U/l, which was not correlated with the concentration of spermatozoa. Most seminal parameters [(\%); total $(77 \pm 2.3)$ and progressive $(62.7 \pm 3.4)$ motility, live sperm $(91.8 \pm 1.2)$, intact plasmalemma (83.5 \pm 2.6$)$, normal acrosomes $(83.5 \pm 2.6), \mathrm{pH}(6.6 \pm 0.0)$ and osmolarity $(\mathrm{mOsm} / \mathrm{l} ; 321 \pm 5.2)$ ], though decreasing during storage in the cold, remained within values compatible with in vivo fertilization for 2 days.
\end{abstract}

Accepted: 16 September 2013

\section{Introduction}

As the domestic cat (Felis catus) is one of the most popular pets worldwide, simple and economically affordable assisted-reproduction technologies become necessary for routine feline veterinary practice. Nevertheless, certain essential aspects of male reproduction have not yet been described in this species.

Because few domestic cats are thought to be trainable in mounting and ejaculating into an artificial vagina $(\mathrm{AV})$, electroejaculation $(\mathrm{EE})$ has been routinely used to collect semen in intact cats. That EE of anesthetized cats causes acute stress as has been demonstrated by an elevation in serum cortisol concentrations immediately after the procedure. ${ }^{1}$ Furthermore, certain anesthetics can affect the sperm quality, ${ }^{2}$ and EE-obtained seminal samples have lower sperm counts and sperm motility than those collected by paraphysiologic methods, for example, AV ejaculation. ${ }^{3,4}$ In view of the implications with respect to feline welfare, non-invasive alternatives to EE should be better explored in such a popular domestic animal. Nevertheless, the success rate of AV ejaculation training has not yet been reported in companion cats.

Alkaline phosphatase (AP) - an enzyme that catalyzes the transport of phosphate groups and that is present in the spermatozoa involved in the fertilization process - has been found in seminal plasma from numerous species.5,6 In cats, as in dogs, as AP originates from the epididymis and testicle, ${ }^{7}$ the

\footnotetext{
1'Laboratory of Reproductive Physiology, Faculty of Veterinary Medicine, National University of La Plata, La Plata, Argentina ${ }^{2}$ Central Laboratory Service, Faculty of Veterinary Medicine, National University of La Plata, La Plata, Argentina
}

\section{Corresponding author:}

Cristina Gobello MV, DMV, Sm An Specialist, DECAR, Laboratory of Reproductive Physiology, Faculty of Veterinary Medicina National University of La Plata 60 \& 118, La Plata, 1900, Argentina Email: cgobello@fcv.unlp.edu.ar, cristinagobello@gmail.com 
enzyme's activity could be used as a marker for tubular patency within the ductal network, as well as an indirect indicator of germ-cell function. Information on seminal AP activity in cats is limited, however, as the concentrations have not been measured in whole ejaculates.

Semen can be diluted in extenders, cooled and maintained at $4^{\circ} \mathrm{C}$ for several days. Extenders protect the spermatozoa, promoting the conservation of motility and fertility over time by stabilizing the plasmalemma, providing energy substrates, and preventing the deleterious effects of progressive changes in $\mathrm{pH}$ and osmolarity. ${ }^{8}$ Seminal storage in the cold can both replace the transport of breeding animals and facilitate dispersion of varied and superior genetic specimens within the felids. In this regard, most of the published preservation studies involve either epididymal ${ }^{9-11}$ or EE-ejaculated ${ }^{11-13}$ semen. Therefore, low-cost, practical and efficient cold-storage diluents that could be used in clinical settings should be tested on AV-ejaculated cat semen.

Milk is a commonly used component of semen extenders in most species, having exhibited good performance both in vitro and in vivo. ${ }^{14,15}$ Skimmed-milk proteins buffer the seminal $\mathrm{pH}$ and may also chelate any heavy metals. ${ }^{14-16}$ The addition of egg yolk to skimmedmilk extenders further improves the viability of spermatozoa during cold storage, ${ }^{14}$ as the yolk phospholipids provide protection to sperm and acrosomal membranes against cold shock. ${ }^{17}$

With the aim of contributing to feline welfare and reproduction, the objectives of this study were to describe (1) domestic cat training in ejaculation into an $\mathrm{AV}$; (2) the AP concentrations in whole ejaculates obtained by an $\mathrm{AV}$; and (3) the in vitro effect of a skimmed-milk with egg yolk (SM-Y) extender on feline spermatozoa incubated at $4^{\circ} \mathrm{C}$.

\section{Materials and methods}

\section{Animals}

In the first experiment, five post-pubertal 5- to 11-month-old mixed-breed male cats were incorporated into our institutional cat colony at the School of Veterinary Medicine, National University of La Plata, Argentina; housed in individual cages $(0.80 \times 0.80 \times$ $1.0 \mathrm{~m}$ ); exposed to a $10 \mathrm{~h}$ dark, $14 \mathrm{~h}$ light photoperiod; fed commercial cat food; and given water ad libitum.

For the second and third experiments, four fertile mixed-breed male cats, born in our cat colony and aged 1-4 years were maintained as in the first experiment. The males were trained to ejaculate into an $\mathrm{AV}$ as described below. These studies were approved by the Faculty Institutional Care and Animal Use Committee.

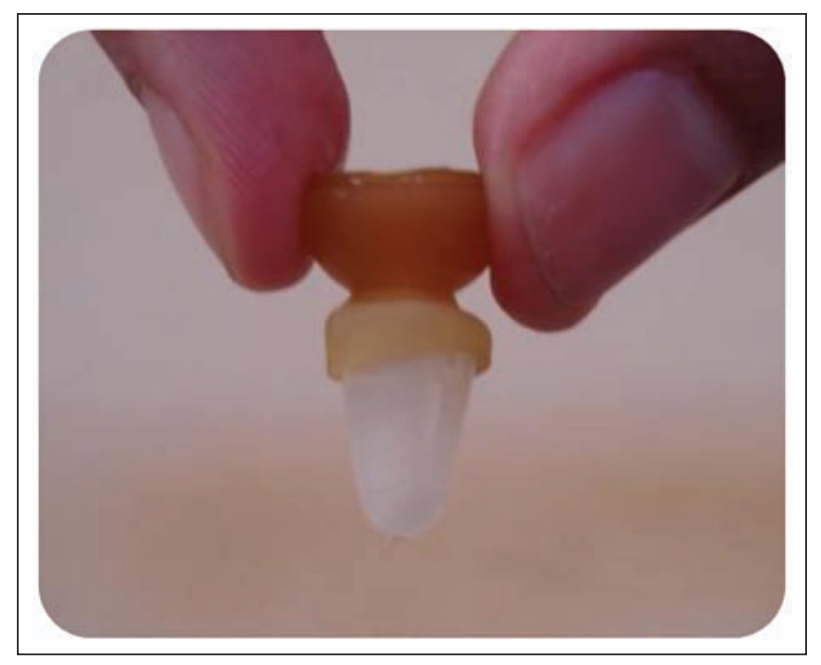

Figure 1 Feline artificial vagina with a semen sample

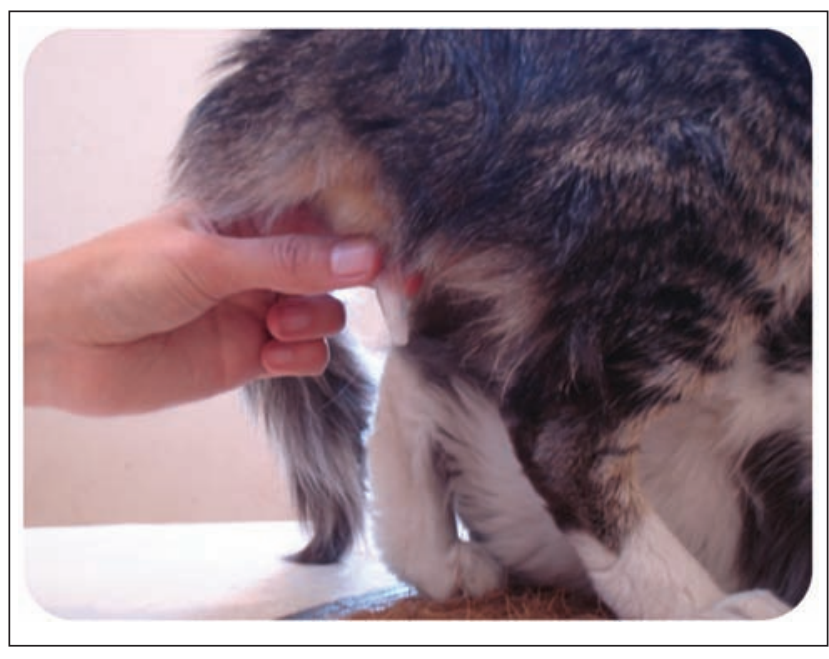

Figure 2 Erect cat penis from which semen has been recently collected by an artificial vagina

\section{Experiment 1}

The cats were trained to ejaculate into an $A^{16}$ in response to manual manipulation of the genitals ${ }^{3}$ three times a week for 20 mins in the presence of a teaser queen until the first ejaculation was obtained. Docile queens in our colony, which were in anestrus, interestrus or estrus, were used for this purpose. The AV was made from a $2 \mathrm{ml}$ rubber pipette bulb and a small test tube (Figure 1 ) as previously described. ${ }^{16}$ The AV was slipped over the erect penis as the cat mounted the queen (Figure 2).

\section{Experiment 2}

Semen samples were collected twice a week repeating the procedure two times with the same AV as described 
in the first experiment. Fifty ejaculates were macro- and microscopically assessed to assure the inclusion of normal specimens.

Color was recorded and the volume measured with a variable micropipette. A drop of semen was placed on a warmed glass slide and the percentage of sperm with total and forward progressive motility subjectively assessed by light microscopy at a magnification of 200x. Vigor was estimated according to an established scale from 0 (absence of movement) to 5 (vigorous movement). The number of sperm was counted by an improved Neubauer hemacytometer chamber. The presence of morphologically normal sperm and sperm with head, midpiece and tail abnormalities were determined by smearing a drop of semen on a glass slide, leaving the slide to dry, staining with Giemsa and examining more than 100 sperm under bright-field microscopy at 1000×. The percentages of live and dead spermatozoa were determined by staining a smear with eosin-nigrosin and examination for eosin uptake at 1000x. Membrane integrity was assessed by the hypo-osmotic swelling (HOS) test ${ }^{18}$ and acrosome integrity after Pope et al. ${ }^{19}$ The $\mathrm{pH}$ was measured with a $\mathrm{pH}$ meter (pH-009; ATC) and the osmolality by an osmometer (model 5520; Wescor).

AP was assayed in duplicate in an autoanalyzer (Metrolab plus 1600) by an optimized colorimetric method (Cod 1361003; Weiner) after dilution of sperm suspensions with physiologic saline (approximately 1:100).

\section{Experiment 3}

Eighty ejaculates were collected, as described above, three times a week. Semen specimens that showed a sperm motility of $80 \%$ and normal morphology of $\geq 70 \%$ were used (day -1$)$. Samples were pooled and diluted (day 0 ) in a test tube (final dilution, 1:3-1:6 with concentrations of $75-100 \times 10^{6} \mathrm{sperm} / \mathrm{ml}$ ) in a SM-Y extender containing $80 \%(\mathrm{v} / \mathrm{v})$ skimmed milk $(0 \%$ fat, ultra high temperature; Ilolay), 20\% (v/v) egg yolk and antibiotics $(1 \mathrm{mg} / \mathrm{ml}$ benzylpenicillin $1 \mathrm{mg} / \mathrm{ml}$ dihydrostreptomycin sulfate). The tube was placed at $4^{\circ} \mathrm{C}$ during the study period in a glass container filled with water to prevent both cold shock and temperature variations during the chilling down and cold storage. Samples were analyzed daily for total and progressive motility, the percentage of live versus dead spermatozoa, normal acrosomes, membrane integrity (HOS), osmolarity and $\mathrm{pH}$, as described for the second experiment.

\section{Statistical analysis}

In the first experiment, the proportion of trained cats, the interval to the first $\mathrm{AV}$ ejaculation and the final resulting performance (defined as libido at semen collection: excellent, good or poor) were recorded and statistically described. Semen parameters were calculated in the
Table 1 Feline seminal parameters (mean \pm SEM) obtained by an artificial vagina

\begin{tabular}{ll} 
Parameter & Mean \pm SEM \\
\hline Color & White opalescent \\
Volume $(\mu \mathrm{l})$ & $102.6 \pm 13.9$ \\
Total motility $(\%)$ & $90.9 \pm 1.0$ \\
Progressive motility (\%) & $88.6 \pm 0.9$ \\
Vigor (0-5) & $4.7 \pm 0.1$ \\
Concentration (10\%/ml) & $450.7 \pm 58.2$ \\
Live sperm (\%) & $88.6 \pm 1.3$ \\
Hypo-osmotic positive (\%) & $90.5 \pm 1.5$ \\
Morpho-anomalies (\%) & 10 \\
Head & 4 \\
Intermediate piece & 2.3 \\
Tail & 3.6 \\
Acrosomal integrity (\%) & $97.3 \pm 0.9$ \\
pH & $7.9 \pm 0.1$ \\
Osmolality (mOsm/l) & $318.7 \pm 7.5$ \\
Alkaline phosphatase (UI/l) & $20,645.6 \pm 4405.4$ \\
\hline
\end{tabular}

second experiment and the correlation between the AP activities and the spermatozoa concentrations analyzed by the Spearman test. In the third experiment, the time course of semen parameters throughout the experiment was analyzed by the repeated-measures analysis of variance. The data were expressed as the means \pm SEM, and $P$-values $<0.05$ considered significant in all instances (SPSS 15.0 software).

\section{Results}

In the first experiment, all the animals (5/5) could be trained to ejaculate, although the interval to the first $\mathrm{AV}$ ejaculation varied from 1.5 to 5.5 months (mean, 3.9 months). The final performance at collection ranged from excellent $(n=1)$ to poor $(n=1)$, and was inversely related to the required training period in all the probands.

The characteristics of the feline semen obtained through the use of an $\mathrm{AV}$ in the second experiment were normal (Table 1). A low, non-significant correlation was found between the AP activities and the concentration of spermatozoa.

In the third experiment, the total $(P<0.01$; Figure 3$)$ and progressive motility $(P<0.01$; Figure 3 , inset), along with the live sperm count $(P<0.05)$, positive endosmosis $(P<0.01)$ and percent intact acrosomes $(P<0.01)$, decreased throughout the experiment, while the $\mathrm{pH}$ and osmolarity underwent no significant change $(P>0.05)$ during the experimental period (Table 2).

\section{Discussion}

This study describes three essential aspects of male cat physiology and reproductive management that have not 


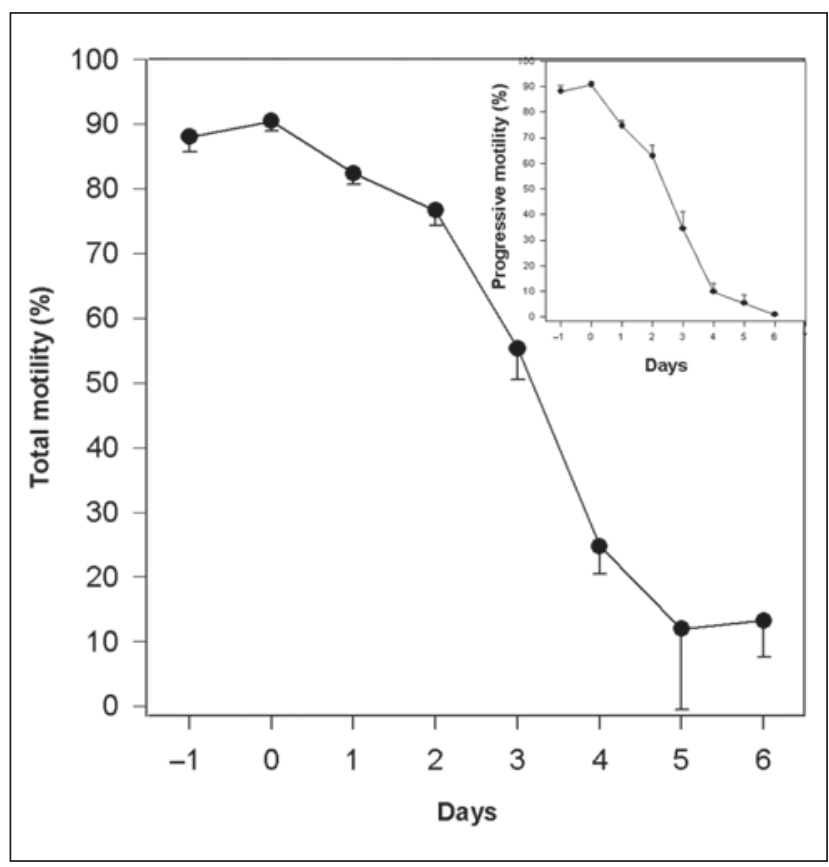

Figure 3 Percentage of total $(P<0.01)$ and progressive $(P<0.01$; inset) motility (mean \pm SEM) of feline semen diluted in a milk-egg yolk extender and stored at $4^{\circ} \mathrm{C}$ during 6 days of observation. Day -1 and day 0 represent the seminal characteristics before and immediately after dilution, respectively

been reported to date. These results could be applied in practice to contribute positively to the welfare and reproductive performance of breeding cats.

Although the number of animals studied was low, according to the results obtained, all domestic cats would appear to be trainable in ejaculating into an AV, though, admittedly, with different final performances, thus exemplifying a situation similar to that observed with other domestic animals. In contrast, a successful collection of semen from $3 / 5(60 \%)$ male cats after 2 weeks of frequent handling has been reported. ${ }^{17}$ The difference in the findings of the present study - the failure to train two of the animals in the previous investigation $^{17}$ - could be explained by the short training period used. Although, in some animals, the training period required by the present protocol can be quite long (up to 5.5 months in this study), the procedure employed would be valid for purebred breeding toms upon consideration of the high quality of the semen samples obtained and the lack of invasiveness of the technique.

In the second experiment the characteristics of the semen were consistent with those previously described for AV semen collection in the species. ${ }^{3,20,21}$ As the felid physiology is such that cats ejaculate in a single fraction, a knowledge of the total AP concentrations in the whole ejaculate will be useful for seminal analysis interpretation. In this experiment, the AP concentrations were within the values previously reported for seminal plasma $(160,355 \pm 15,558 \mathrm{U} / \mathrm{l})$ and for prostatic (445 \pm $179 \mathrm{U} / \mathrm{l})$ and bulbourethral fluids $(281 \pm 164) .{ }^{7}$ Despite this agreement, in cats, AP originates in the epididymides and testes, ${ }^{7}$ but the total levels in the present ejaculates did not seem to be related to the semen concentration, at least in these normal animals. Whether different grades of oligozoospermia could be correlated with seminal AP concentrations remains to be determined.

The SM-Y chilling extender conserved the feline AV-obtained semen in an acceptable condition for subsequent in vivo fertilization for up to 2 days. In this trial, however, motility had to be subjectively estimated as computerized techniques cannot be used with milkbased extenders. This limitation was compensated for by having each sample evaluated by two trained observers. The other in vitro seminal parameters remained within the normal ranges for a further 1 or 2 days. These in vitro results would identify SM-Y as constituting a low-cost, efficient and practical feline semen extender.

Table 2 Characteristics (mean \pm SEM) of feline semen diluted in a milk-egg yolk extender and stored at $4^{\circ} \mathrm{C}$ during 6 days of observation

\begin{tabular}{llllll} 
Day & Live sperm $(\%)^{*}$ & $\begin{array}{l}\mathrm{HOS} \\
(\%)^{\star *}\end{array}$ & $\mathrm{pH}$ & $\begin{array}{l}\text { Intact } \\
\text { acrosome }(\%)^{\star *}\end{array}$ & Osm (mOsm/l) \\
\hline $\mathbf{0}^{\dagger}$ & $93.9 \pm 1.1$ & $92.0 \pm 0.8$ & $6.4 \pm 0.1$ & $95.0 \pm 1.5$ & $318.0 \pm 13.5$ \\
$\mathbf{1}$ & $93.8 \pm 0.9$ & $89.3 \pm 0.5$ & $6.5 \pm 0.0$ & $90.58 \pm 1.10$ & $318.0 \pm 7.5$ \\
$\mathbf{2}$ & $91.8 \pm 1.2$ & $83.5 \pm 2.6$ & $6.6 \pm 0.0$ & $83.41 \pm 2.70$ & $321.0 \pm 5.2$ \\
$\mathbf{3}$ & $90.0 \pm 5.8$ & $83.1 \pm 1.8$ & $6.6 \pm 0.0$ & $78.9 \pm 3.4$ & $325.0 \pm 6.0$ \\
$\mathbf{4}$ & $84.4 \pm 3.3$ & $71.5 \pm 2.4$ & $6.7 \pm 0.0$ & $66.5 \pm 8.5$ & $319.0 \pm 15.6$ \\
$\mathbf{5}$ & $83.2 \pm 3.6$ & $70.5 \pm 4.5$ & $6.6 \pm 0.0$ & 53.2 & $327.6 \pm 10.0$ \\
$\mathbf{6}$ & $74.7 \pm 5.6$ & $71.2 \pm 2.1$ & $6.7 \pm 0.0$ & 40.0 & $327.2 \pm 7.5$
\end{tabular}

${ }^{\star} P<0.05 ;{ }^{* *} P<0.01$

tDay 0 represents the seminal characteristics immediately after dilution. HOS = hypo-osmotic swelling 
Furthermore, cat semen could be cooled in this milkand egg-yolk-based diluent in clinics for subsequent transport to semen banks for freezing. The assessment of pregnancy rates after artificial insemination with these diluted, chilled and frozen-thawed samples, however, would have to be undertaken before such semen preparations could be widely indicated for that purpose.

\section{Conclusions}

All domestic cats can be trained to ejaculate into an AV after a variable training period. An AV-obtained semen has a mean AP of 20,645 U/1 and samples acquired in this manner can be successfully preserved at $4^{\circ} \mathrm{C}$ in an SM-Y extender for up to 2 days.

Funding This study was partially funded University Incentive Program for Teaching and Research (V 195 to CG).

Conflict of interest The authors do not have any potential conflicts of interest to declare.

\section{References}

1 Carter KK, Chakraborty PK, Bush M, et al. Effects of electroejaculation and ketamine-HCI on serum cortisol, progesterone, and testosterone in the male cat. J Androl 1984; 5: 431-437.

2 Jiménez E, Pérez-Marín CC, Millán Y, et al. Influence of anaesthetic drugs on the epididymal sperm quality in domestic cats. Anim Reprod Sci. 2011; 123: 265-269.

3 Platz CC, Wildt DE and Seager SW. Pregnancy in the domestic cat after artificial insemination with previously frozen spermatozoa. J Reprod Fertil 1978; 52: 279-282.

4 Dooley MP and Pineda MH. Effect of method of collection on seminal characteristics of the domestic cat. Am J Vet Res 1986; 47: 286-292.

5 Lewis-Jones DI, Johnson PM, Desmond AD, et al. Germ cell alkaline phosphatase in human seminal plasma following vasectomy. Br J Urol 1992; 69: 418-420.

6 Gobello C, Castex G and Corrada Y. Serum and seminal markers in the diagnosis of disorders of the genital tract of the dog: a mini-review. Theriogenology 2002: 57: 1285-1291.
7 Johnston SD, Osborne CA and Lipowitz AJ. Characterization of seminal plasma, prostatic fluid and bulbourethal gland secretions in the domestic cat. In: Proceedings 11th international congress on animal reproduction and artificial insemination, vol. IV, Dublin, Ireland, 1988, p 560.

8 Linde-Forsberg C. Artificial insemination with fresh, chilled, extended and frozen-thawed semen in the dog. Semin Vet Med Surg (Small Anim) 1992; 10: 48-58.

9 Goodrowe KL and Hay MA. Characteristics and zona binding ability of fresh and cooled domestic cat spermatozoa. Theriogenology 1993; 40: 967-975.

10 Hermansson U and Axnér E. Epididymal and ejaculated cat spermatozoa are resistant to cold shock but egg yolk promotes sperm longevity during cold storage at 4 degrees C. Theriogenology 2007; 67: 1239-1248.

11 Harris RF, Pope CE, Gomez MC, et al. Storage of domestic cat spermatozoa for extended periods at $4^{\circ} \mathrm{C}$. Theriogenology 2001; 55: 308-308.

12 Glover TE and Watson PF. The effect of buffer osmolality on the survival of cat (Felis catus) spermatozoa at $5^{\circ} \mathrm{C}$. Theriogenology 1985; 24: 449.

13 Glover TE and Watson PF. The effects of egg yolk, the low density lipoprotein fraction of egg yolk, and three monosaccharides on the survival of cat (Felis catus) spermatozoa stored at $5^{\circ} \mathrm{C}$. Anim Reprod Sci 1987; 13: 229-237.

14 Maxwell W and Salamon S. Liquid storage of ram semen: a review. Reprod Fertil Dev 1993; 5: 613-638.

15 Batellier F, Vidament M, Fauquant J, et al. Advances in cooled semen technology. Anim Reprod Sci 2001; 68; 181-190.

16 Sojka NJ, Jemings LL and Hamner CE. Artificial insemination in the cat (Felis catus). Lab Anim Care 1970; 20: 198-204.

17 Jones RC and Martin ICA. The effects of dilution, egg yolk and cooling to $5^{\circ} \mathrm{C}$ on the ultrastructure of ram spermatozoa. J Reprod Fertil 1973; 35: 311-320.

18 England G and Plummer J. Hipoosmotic swelling of dog spermatozoa. J Reprod Fertil (Suppl) 1993; 47: 261-270.

19 Pope CE, Zhang YZ and Dresser BL. A simple staining method for evaluating acrosomal status of cat spermatozoa. J Zoo Wildl Med 1991; 22: 87-95.

20 Zambelli D and Cunto M. Semen collection in cats: techniques and analysis. Theriogenology 2006; 66: 159-165.

21 Tanaka A, Takagi Y, Nakagawa K, et al. Artificial intravaginal insemination using fresh semen in cats. J Vet Med Sci 2000; 62: 1163-1167. 